\title{
A generating family for the Freudenthal compactification of a class of rimcompact spaces
}

\author{
by
}

\author{
Jesús M. Domínguez (Valladolid)
}

\begin{abstract}
For $X$ a Tikhonov space, let $F(X)$ be the algebra of all real-valued continuous functions on $X$ that assume only finitely many values outside some compact subset. We show that $F(X)$ generates a compactification $\gamma X$ of $X$ if and only if $X$ has a base of open sets whose boundaries have compact neighborhoods, and we note that if this happens then $\gamma X$ is the Freudenthal compactification of $X$. For $X$ Hausdorff and locally compact, we establish an isomorphism between the lattice of all subalgebras of $F(X) / C_{\mathrm{K}}(X)$ and the lattice of all compactifications of $X$ with zero-dimensional remainder, the finite-dimensional subalgebras corresponding to the compactifications with finite remainder.
\end{abstract}

Introduction. Throughout the paper $C(X)$ will stand for the algebra of all real-valued continuous functions on a non-empty Tikhonov space $X$, and $C^{*}(X)$ for the subalgebra of bounded functions. $C^{\#}(X)$ will be the subset of $C(X)$ consisting of all $f$ with the property that, for every maximal ideal $M$ of $C(X)$, there exists $\lambda_{M} \in \mathbb{R}$ such that $f-\lambda_{M} \in M$. We shall denote by $F(X)$ the subset of $C(X)$ consisting of all $f$ such that $f(X-K)$ is a finite set for some compact $K \subseteq X, K$ depending on $f$. Both $C^{\#}(X)$ and $F(X)$ are subalgebras of $C^{*}(X)$, and $F(X) \subseteq C^{\#}(X)$. Moreover, $F(X)=C^{\#}(X)$ for $X$ realcompact and locally compact (see $[20]$ ).

The space $X$ is said to be rimcompact if it has a base of open sets with compact boundaries. Each rimcompact space has a compactification $\phi X$ that is the maximum compactification of $X$ with zero-dimensional remainder; $\phi X$ is called the Freudenthal compactification of $X$.

Henriksen was the first to observe the relationship between $C^{\#}(X)$ and $\phi X$; he showed in [12] that if $X$ is realcompact and $C^{\#}(X)$ generates a

2000 Mathematics Subject Classification: 54D35, 54C40.

Key words and phrases: compactification, rimcompact, ring of continuous functions, function algebra, Boolean ring, maximal ideal, idempotent, zero-dimensional.

Partially supported by DGICYT grant BFM2002-04125-C02-01. 
compactification of $X$, then $X$ is rimcompact and $\phi X$ is the compactification generated by $C^{\#}(X)$. Ünlü proved in [21] that $F(X)$ generates $\phi X$ if $X$ is locally compact. Here we show that $F(X)$ generates a compactification of $X$ if and only if $X$ has a base of open sets whose boundaries have compact neighborhoods, and we note that in that case the compactification is $\phi X$. The question of exactly when $C^{\#}(X)$ generates $\phi X$ remains open.

Assume now that $X$ is a locally compact space. We shall denote by $C_{\infty}(X)$ the ideal of $C^{*}(X)$ consisting of all functions in $C(X)$ that vanish at infinity, and by $C_{\mathrm{K}}(X)$ the ideal of all continuous functions with compact support. If $A$ is a closed subalgebra of $C^{*}(X)$ containing $C_{\infty}(X)$, then the maximal ideal space $\operatorname{Max} A$ of $A$ is a compactification of $X$, and every compactification of $X$ can be obtained this way. The correspondence $A \mapsto \operatorname{Max} A$ establishes an isomorphism between the lattice of closed subalgebras of $C^{*}(X)$ that contain $C_{\infty}(X)$, and the lattice $\mathcal{K}(X)$ of all compactifications of $X$.

Magill proved in [16] that, for $X$ and $Y$ locally compact spaces, $\mathcal{K}(X)$ and $\mathcal{K}(Y)$ are isomorphic lattices if and only if $\beta X-X$ and $\beta Y-Y$ are homeomorphic spaces. Let us denote by $\mathcal{K}_{0}(X)$ the lattice of all compactifications of $X$ with zero-dimensional remainder. We show that $F(X)$ and $C_{\mathrm{K}}(X)$ play with respect to $\mathcal{K}_{0}(X)$ a similar role to that of $C^{*}(X)$ and $C_{\infty}(X)$ in the case of $\mathcal{K}(X)$. We establish an isomorphism between $\mathcal{K}_{0}(X)$ and the lattice of all subalgebras of $F(X) / C_{\mathrm{K}}(X)$, and we make use of this isomorphism to derive the zero-dimensional version of Magill's theorem (Woods [23]): "For $X$ and $Y$ locally compact spaces, $\mathcal{K}_{0}(X)$ and $\mathcal{K}_{0}(Y)$ are isomorphic lattices if and only if the remainders $\phi X-X$ and $\phi Y-Y$ are homeomorphic spaces".

Section 1 contains the algebraic treatment. By making abstraction of the algebraic properties of $F(X) / C_{\mathrm{K}}(X)$, we study the algebras over a fixed field that are linearly generated by their idempotents; we call them LC-algebras, as they are algebras of locally constant functions. In Section 2 we prove that $F(X)$ generates a compactification of $X$ if and only if $X$ has a base of open sets whose boundaries have compact neighborhoods. Finally, in Section 3 we establish the lattice isomorphisms, and we derive the zero-dimensional version of Magill's theorem.

1. Algebraic treatment. For algebraic concepts we shall basically adhere to the notation and terminology in [1]. The word "ring" will always mean a commutative ring with identity element 1 , the subrings of a ring $A$ contain the identity element of $A$, and the ring morphisms preserve the identity elements. From now on $A$ will be a ring.

We now review some definitions and results that will be used throughout the paper. 
1.1. The prime and maximal spectra, $\operatorname{Spec} A$ and $\operatorname{Max} A$. By $\operatorname{Spec} A$ we mean the prime spectrum of the ring $A$, that is, the set of all prime ideals of $A$ with the Zariski (or hull-kernel) topology. The sets $V(F)=\{P \in$ Spec $A: F \subseteq P\}$, where $F \subseteq A$, are the closed subsets of $\operatorname{Spec} A$. We shall denote by Max $A$ the maximal spectrum of $A$, that is, the subspace of Spec $A$ consisting of all maximal ideals of $A$. For $F \subseteq A$, we shall write $V^{M}(F)=V(F) \cap \operatorname{Max} A$.

Let $\psi: A \rightarrow B$ be a ring morphism. If $Q$ is a prime ideal of $B$, then $\psi^{-1}(Q)$ is a prime ideal of $A$, so that $\psi$ induces a continuous map

$$
\psi^{*}: \operatorname{Spec} B \rightarrow \operatorname{Spec} A, \quad Q \mapsto \psi^{-1}(Q) .
$$

In this way one establishes a contravariant functor from the category of rings to the category of topological spaces.

Let $I$ be an ideal of $A$. The canonical map $A \rightarrow A / I$ induces a topological embedding $\operatorname{Spec}(A / I) \rightarrow \operatorname{Spec} A$ whose image is $V(I)$. This embedding allows us to identify $\operatorname{Spec}(A / I)$ with $V(I)$. We also identify $\operatorname{Max}(A / I)$ with $V^{M}(I)$.

We shall say that $A$ is a reduced ring if $\bigcap \operatorname{Spec} A=\{0\}$, and $A$ will be called semisimple if $\bigcap$ Max $A=\{0\}$.

We shall denote by $A_{P}$ the localization of the $\operatorname{ring} A$ at the prime ideal $P$; in other words, $A_{P}=S^{-1} A$, where $S=A-P$.

Recall that a ring $A$ is regular (in the sense of von Neumann) or absolutely flat if, for every $x \in A$, there exists $x^{\prime} \in A$ such that $x x^{\prime} x=x$.

The next result is well known (see [1]).

1.2. TheOrem. For a ring A, the following conditions are equivalent:

(a) $A$ is a regular ring.

(b) Each principal ideal of $A$ is generated by an idempotent.

(c) $A$ is a reduced ring and every prime ideal of $A$ is maximal.

(d) For every $M \in \operatorname{Max} A$, the local ring $A_{M}$ is a field.

If $A$ is a regular ring, then Max $A$ is a Boolean space, that is, a compact Hausdorff and zero-dimensional space.

1.3. The Boolean ring $B(A)$. We shall denote by $B(A)$ the set of all idempotents of an arbitrary ring $A$. We keep the product inherited from $A$, and we define a new addition in $B(A)$ by the rule:

$$
e \star f=e+f-2 e f \quad \text { for } e, f \in B(A) .
$$

With these operations $B(A)$ is a Boolean ring.

1.4. Locally constant functions. From now until the end of Section 1, $E$ will be an infinite field, and the word "algebra" will mean a non-zero $E$-algebra. If $A$ is an algebra, we shall identify each element of $E$ with its image in $A$ under the structural morphism $E \rightarrow A$. For $M$ a maximal ideal 
of $A$, we shall write $A / M=E$ (resp. $A_{M}=E$ ) if the structural morphism $E \rightarrow A / M$ (resp. $E \rightarrow A_{M}$ ) is an isomorphism.

We adapt the notation and some of the results in [15]. For $X$ a compact Hausdorff space, we shall denote by $\operatorname{LC}(X)$ the algebra of all locally constant functions from $X$ to $E$. The idempotents of $\operatorname{LC}(X)$ are the characteristic functions, with values in $E$, of the clopen (both open and closed) subsets of $X$. Every element of $\operatorname{LC}(X)$ is a linear combination of idempotents.

1.5. $L C$-algebras. We shall say that an algebra $A$ is an $L C$-algebra if every $f \in A$ is a linear combination of idempotents, that is, if

$$
f=\sum_{i=1}^{n} \lambda_{i} e_{i}, \quad \text { where } \lambda_{i} \in E, e_{i} \in B(A), \text { for } 1 \leq i \leq n .
$$

We shall say that an algebra $A$ is a Gelfand algebra if, for every maximal ideal $M$ of $A$, one has $A / M=E$, that is, for every $f \in A$ and every $M \in \operatorname{Max} A$, there exists $\lambda \in E$ such that $f-\lambda \in M$.

Let us consider $E^{n}=E \times \ldots \times E$ with the sum and product operations defined componentwise. The map $E \rightarrow E \times \ldots \times E, \lambda \mapsto(\lambda, \ldots, \lambda)$, defines on $E^{n}$ an $E$-algebra structure. In fact, $E^{n}$ is an LC-algebra.

Let $A \subseteq B$ be a ring extension. Recall that an element $b \in B$ is said to be integral over $A$ if $b$ is a root of a monic polynomial $P(x)=x^{n}+a_{n-1} x^{n-1}+$ $\ldots+a_{0}$ with coefficients in $A$. The ring $B$ is said to be integral over $A$ if every element of $B$ is integral over $A$.

The next result can be easily proved, and so we shall not include its proof.

1.6. Theorem (see [15] and compare with Th. 1.2). For an algebra A, the following conditions are equivalent:

(a) $A$ is an LC-algebra.

(b) $A$ is a reduced algebra and for every $f \in A$ there exist $\lambda_{1}, \ldots, \lambda_{n}$ in $E$ such that

$$
\prod_{i=1}^{n}\left(f-\lambda_{i}\right)=0
$$

(c) $A$ is a semisimple Gelfand algebra and an integral extension of $E$.

(d) $A$ is isomorphic to $\operatorname{LC}(X), X$ being a Boolean space.

(e) $A_{M}=E$ for every $M \in \operatorname{Max} A$.

It follows from the above theorem that LC-algebras are regular rings, and so their maximal spectra are Boolean spaces. It is clear that quotients by a proper ideal and subalgebras of LC-algebras are also LC-algebras. It is also clear that a finite tensorial product or an inductive limit of LC-algebras (in the category of $E$-algebras) is again an LC-algebra. Therefore, a tensorial product of LC-algebras (in the category of $E$-algebras) is an LC-algebra. It 
follows from the preceding theorem that a finite product of LC-algebras (in the category of $E$-algebras) is again an LC-algebra.

1.7. The Gelfand transformation and the spectral representation. Let $A$ be an LC-algebra. Since $A$ is a Gelfand algebra, we can associate to every $f \in A$ the function $\widehat{f}: \operatorname{Max} A \rightarrow E, M \mapsto M(f)$, where $M(f)$ is the residue class of $f$ modulo the maximal ideal $M$. The Gelfand transformation is the map

$$
G_{A}: A \rightarrow \operatorname{LC}(\operatorname{Max} A), \quad f \mapsto \widehat{f} .
$$

It is an algebra isomorphism which is functorial in $A$ (see [15]).

Let $X$ be a Boolean space. For $x \in X$, set $M_{x}=\{f \in \mathrm{LC}(X): f(x)=0\}$. The spectral representation

$$
\mu_{X}: X \rightarrow \operatorname{Max} \operatorname{LC}(X), \quad x \mapsto M_{x},
$$

is a homeomorphism which is functorial in $X$.

The duality between Boolean lattices, Boolean rings and Boolean spaces is well known. We shall see that this duality may be extended to the LCalgebras.

Let $\mathcal{B S}$ be the category of Boolean spaces (and continuous maps), set $\mathcal{L C}$ for the category of LC-algebras (and $E$-algebra morphisms), and denote by $\mathcal{B S}^{\text {op }}$ the opposite category to $\mathcal{B S}$.

1.8. TheOREM. The category $\mathcal{L C}$ is equivalent to the category $\mathcal{B S}^{\text {op }}$.

Proof. On the one hand, a continuous map $\alpha: X \rightarrow X^{\prime}$ between Boolean spaces induces, by composition, a morphism of algebras $\mathrm{LC}\left(X^{\prime}\right) \rightarrow \mathrm{LC}(X)$, $f \mapsto f \circ \alpha$, so that LC is a contravariant functor from $\mathcal{B S}$ into $\mathcal{L C}$. On the other hand, Max is a contravariant functor from $\mathcal{L C}$ into $\mathcal{B S}$. The Gelfand transformation $\left(G_{-}\right)$and the spectral representation $\left(\mu_{-}\right)$establish the needed functorial isomorphisms.

1.9. EXAMPLE. Let $S$ be the algebra of all periodic sequences with values in the field $E$, and denote by $P$ the set of all positive prime integers.

For $p \in P$, set

$$
S_{p}=\left\{f \in S: p^{n} \text { is a period of } f \text { for some } n \in \mathbb{N}\right\},
$$

and, for $n \in \mathbb{N}$, set

$$
S\left(p^{n}\right)=\left\{f \in S: p^{n} \text { is a period of } f\right\} .
$$

$S_{p}$ is the inductive limit of the subalgebras $S\left(p^{n}\right)$, and $S$ is the tensorial product of the subalgebras $S_{p}$, that is,

$$
S_{p} \cong \lim _{n \in \mathbb{N}} S\left(p^{n}\right), \quad S \cong \bigotimes_{p \in P} S_{p}
$$

The algebras $S\left(p^{n}\right), S_{p}$, and $S$ are all LC-algebras. 
According to 1.8 ,

$$
\operatorname{Max} S_{p} \cong \lim _{n \in \mathbb{N}} \mathbb{Z} /\left(p^{n}\right) \cong \mathbb{Z}_{p}, \quad \operatorname{Max} S \cong \prod_{p \in P} \operatorname{Max} S_{p} \cong \prod_{p \in P} \mathbb{Z}_{p},
$$

where the quotient ring $\mathbb{Z} /\left(p^{n}\right)$ has the discrete topology, and $\mathbb{Z}_{p}$ is the ring of the $p$-adic integers with the topology induced by the $p$-adic absolute value.

Both the product $\prod_{p \in P} \mathbb{Z}_{p}$ and each factor $\mathbb{Z}_{p}$ are perfect compact metric spaces which are zero-dimensional. Hence, they are all homeomorphic to the Cantor ternary set. Thus, $\operatorname{Max} S \cong \operatorname{Max} S_{p}$. It follows from 1.8 that $S \cong S_{p}$ for every $p \in P$. In particular, $S \cong S_{2}$.

Recall that a collection of functions separates points in $X$ if, whenever $x \neq y \in X$, there is a function $f$ in the collection such that $f(x) \neq f(y)$.

1.10. Theorem. If $X$ is a Boolean space and $A$ is a subalgebra of $\mathrm{LC}(X)$ that separates points, then $A=\mathrm{LC}(X)$.

Proof. Since $A$ separates points, the contraction of maximal ideals

$$
X=\operatorname{Max} \mathrm{LC}(X) \rightarrow \operatorname{Max} A, \quad M_{x} \mapsto M_{x} \cap A,
$$

is a continuous and injective mapping between compact Hausdorff spaces. Moreover, it is surjective because $A \subseteq \mathrm{LC}(X)$ is an integral extension of rings (see $[1,5.10]$ ). It is, then, a homeomorphism.

We say that an algebra is finite-dimensional if it is a finite-dimensional vector space over the field $E$.

The algebra $A$ is singly generated if there exists $f \in A$ such that $A=$ $E[f]$, that is, every element of $A$ can be written as a polynomial in $f$ with coefficients in $E$. In that case, $f$ is said to be a primitive element for the extension $E \subseteq A$.

Recall that a singly generated $E$-algebra is finite-dimensional if it is an integral extension of $E$.

1.11. TheOrem. For an LC-algebra A, the following conditions are equivalent:

(a) $A$ is an algebra isomorphic to $E^{n}$ for some $n \in \mathbb{N}$.

(b) $A$ is a finite-dimensional algebra.

(c) $\operatorname{Max} A$ is a finite set.

(d) $A=E[f]$, that is, $A$ has a primitive element.

Proof. The first three conditions are clearly equivalent.

(a) $\Rightarrow(\mathrm{d})$. Set $f=\left(\lambda_{1}, \ldots, \lambda_{n}\right) \in E^{n}$, where $\lambda_{i} \neq \lambda_{j}$ for $i \neq j$. Since $E[f]$ separates points in Max $E^{n}$, it follows from 1.10 that $E[f]=\mathrm{LC}\left(\operatorname{Max} E^{n}\right)$ $=A$.

$(\mathrm{d}) \Rightarrow(\mathrm{b}) . \quad A$ is a finite-dimensional algebra because it is a singly generated $E$-algebra and an integral extension of $E$. 
If $A$ is an LC-algebra (resp. a Boolean ring) we shall denote by $\mathcal{L}(A)$ the lattice of all subalgebras (resp. subrings) of $A$. We shall denote by $\mathcal{L}_{\mathrm{F}}(A)$ the sublattice of $\mathcal{L}(A)$ consisting of all finite-dimensional subalgebras of the LC-algebra $A$. Then $\mathcal{L}_{\mathrm{F}}(A)$ is a lower complete lattice.

We shall not include the proof of the next result.

1.12. Theorem (Sachs [19], Filippov [7]). The Boolean rings $A_{1}$ and $A_{2}$ are isomorphic if and only if $\mathcal{L}\left(A_{1}\right)$ and $\mathcal{L}\left(A_{2}\right)$ are isomorphic lattices.

1.13. Corollary. Let $A_{1}$ and $A_{2}$ be $L C$-algebras. The following conditions are equivalent:

(a) $A_{1}$ and $A_{2}$ are isomorphic algebras.

(b) $\mathcal{L}_{\mathrm{F}}\left(A_{1}\right)$ and $\mathcal{L}_{\mathrm{F}}\left(A_{2}\right)$ are isomorphic lattices.

(c) $\mathcal{L}\left(A_{1}\right)$ and $\mathcal{L}\left(A_{2}\right)$ are isomorphic lattices.

(d) Max $A_{1}$ and Max $A_{2}$ are homeomorphic spaces.

Proof. It is clear that (a) implies (b), and we already know that (a) and (d) are equivalent.

(b) $\Rightarrow(\mathrm{c})$. Let $\phi: \mathcal{L}_{\mathrm{F}}\left(A_{1}\right) \rightarrow \mathcal{L}_{\mathrm{F}}\left(A_{2}\right)$ be a lattice isomorphism. Observe that, for any subalgebra $A$ of $A_{1}$,

$$
A=\bigvee\{C: C \text { is a finite-dimensional subalgebra of } A\} .
$$

We extend $\phi$ to a new lattice isomorphism $\Phi: \mathcal{L}\left(A_{1}\right) \rightarrow \mathcal{L}\left(A_{2}\right)$ by defining

$$
\Phi(A)=\bigvee\{\phi(C): C \text { is a finite-dimensional subalgebra of } A\} .
$$

$(\mathrm{c}) \Rightarrow(\mathrm{a})$. This will follow from 1.12 , once we observe that, for any LCalgebra $A$, the map that sends a subalgebra $C$ of $A$ to the Boolean ring $B(C)$ is a lattice isomorphism between $\mathcal{L}(A)$ and $\mathcal{L}(B(A))$.

1.14. Remark. It is possible to repeat the argument in [16] and prove 1.13 without making use of 1.12 , and then one may obtain 1.12 as a consequence of the duality established in 1.8 .

2. $F(X)$ generates $\phi X$. For topological concepts we shall adhere to the notation and terminology in [8] and [2], but we shall use the word "algebra" as in the first section, with $E=\mathbb{R}$. Therefore, all the subalgebras of $C(X)$ contain the constant functions.

Recall that $X$ is a non-empty Tikhonov space, and $F(X)$ is the algebra of all $f$ in $C(X)$ such that $f(X-K)$ is finite for some compact $K \subseteq X$, $K$ depending on $f$.

Let us describe $F(X)$ in some particular cases. The algebra $F(\mathbb{R})$ consists of those functions in $C(\mathbb{R})$ that are constant on both $(-\infty, a)$ and $(b, \infty)$ for some $a, b \in \mathbb{R}$. For $n \geq 2, F\left(\mathbb{R}^{n}\right)$ consists of those functions in $C\left(\mathbb{R}^{n}\right)$ that are constant on $\left\{x \in \mathbb{R}^{n}:\|x\|>a\right\}$ for some $a \in \mathbb{R}$. If no point 
of $X$ has a compact neighborhood, then $F(X)$ consists of those functions in $C(X)$ with finite range. Therefore, for $X$ an infinite product of copies of $\mathbb{R}$, the only functions in $F(X)$ are the constant ones. $F(\mathbb{N})$ consists of the real sequences with finite range. And finally, if $\mathbb{W}$ is the space of all countable ordinals (with the order topology), then $F(\mathbb{W})=C(\mathbb{W})$.

Recall that a collection of functions separates points from closed sets in $X$ if, for every point $x$ of $X$ and every closed subset $B$ of $X$ not containing $x$, there is a function $f$ in the collection such that $f(x) \notin \mathrm{cl} f(B)$.

Assume that $S$ is a subalgebra of $C^{*}(X)$ and that $\alpha X$ is a compactification of $X$. We shall say that $S$ generates $\alpha X$ if every $f \in S$ has an extension $f^{\alpha} \in C(\alpha X)$ and $S^{\alpha}=\left\{f^{\alpha}: f \in S\right\}$ separates points in $\alpha X$. We shall say that $S$ determines $\alpha X$ if every $f \in S$ has an extension $f^{\alpha} \in C(\alpha X)$ and $S^{\alpha}=\left\{f^{\alpha}: f \in S\right\}$ separates points in $\alpha X-X$. If $S$ generates $\alpha X$, then $S$ determines $\alpha X$. It is well known (see $[22,2.2]$ ) that $S$ generates a compactification of $X$ if and only if it separates points from closed sets in $X$.

Let $C_{\alpha}(X)$ denote the algebra of all functions $f \in C^{*}(X)$ continuously extendable to $\alpha X$. We shall write $F_{\alpha}=F(X) \cap C_{\alpha}(X)$.

We shall denote by $K_{\alpha}(X)$ the closure of $\alpha X-X$ in $\alpha X$. Observe that $K_{\alpha}(X)=(\alpha X-X) \cup R(X)$, where $R(X)$ is the set of points in $X$ that fail to have a compact neighborhood.

2.1. LEMmA. If $S$ is a subalgebra of $F(X)$ that generates a compactification $\alpha X$ of $X$, then $K_{\alpha}(X)$ is zero-dimensional.

Proof. Assume that $S$ generates a compactification $\alpha X$. If $f \in F(X)$ then $f^{\alpha}\left(K_{\alpha}(X)\right)$ is finite, so the finite-valued continuous functions $K_{\alpha}(X) \rightarrow$ $\mathbb{R}$ separate points in $K_{\alpha}(X)$. Thus, $K_{\alpha}(X)$ is zero-dimensional.

It is a known result that there exists a compactification $\alpha X$ with $K_{\alpha}(X)$ zero-dimensional if and only if $X$ has a base of open sets whose boundaries have compact neighborhoods (see [14, VI.29] or [10,3.1]). Note that the last condition is satisfied by every Hausdorff locally compact space, and by every space $X$ that is zero-dimensional at each point of $R(X)$.

2.2. THEOREM. The following statements are equivalent:

(a) $F_{\alpha}(X)$ separates points from closed sets in $X$ for every compactification $\alpha X$.

(b) $F(X)$ separates points from closed sets in $X$, that is, $F(X)$ generates a compactification of $X$.

(c) There exists a compactification $\alpha X$ of $X$ such that $K_{\alpha}(X)$ is zerodimensional.

(d) $X$ has a base of open sets whose boundaries have compact neighborhoods. 
Proof. It is evident that (a) implies (b), it follows from the previous lemma that (b) implies (c), and we already know that (c) and (d) are equivalent. Now we prove that (d) implies (a).

Let $\alpha X$ be a compactification of $X$. We are going to see that $F_{\alpha}(X)$ separates points from closed sets in $X$. Take a closed subset $B$ of $X$ and a point $x \in X-B$. By the hypothesis, there exists an open neighborhood $U$ of $x$ having a compact boundary and such that $B \subseteq \operatorname{int}(X-U)$ and Fr $U \subseteq X-R(X)$. Now, take a compact neighborhood $K$ of $\operatorname{Fr} U$ such that $K \cap B=\emptyset$. One has

$$
\operatorname{cl}_{\alpha X} U \cap \operatorname{cl}_{\alpha X}(X-(U \cup K)) \subseteq \operatorname{cl}_{\alpha X} U \cap \operatorname{cl}_{\alpha X}(X-\operatorname{cl} U) \subseteq \operatorname{Fr} U .
$$

But Fr $U \subseteq \operatorname{int}_{\alpha X} K$, since $K$ is a compact neighborhood of Fr $U$. Hence,

$$
\operatorname{cl}_{\alpha X} U \cap \operatorname{cl}_{\alpha X}(X-(U \cup K)) \subseteq \operatorname{cl}_{\alpha X}(X-K) \cap \operatorname{int}_{\alpha X} K=\emptyset .
$$

Therefore, there exists $f \in C_{\alpha}(X)$ such that

$$
f= \begin{cases}1 & \text { on } \operatorname{cl}_{\alpha X} U \\ 0 & \text { on } \operatorname{cl}_{\alpha X}(X-(U \cup K)) .\end{cases}
$$

Certainly $f \in F_{\alpha}(X)$, since $f(X-K)=\{0,1\}$. Moreover, $f(x)=1$ and $f(B)=\{0\}$ as required.

We omit the proof of the next result since it is completely analogous to the argument in $[12,2.5]$.

2.3. TheOREM. If $F(X)$ generates a compactification of $X$, then $X$ is rimcompact and the compactification is $\phi X$.

\section{The lattice isomorphisms}

3.1. Notation. We shall always identify equivalent compactifications of the non-empty Tikhonov space $X$. We shall denote by $\mathcal{K}(X)$ the complete upper semilattice of all compactifications of $X$. Recall that $\mathcal{K}(X)$ is a complete lattice if and only if $X$ is Hausdorff and locally compact. We shall denote by $\mathcal{K}_{0}(X)$ the subset of $\mathcal{K}(X)$ consisting of all compactifications with zero-dimensional remainder, and by $\mathcal{K}_{\mathrm{F}}(X)$ the subset of those compactifications with finite remainder.

Observe that, for $\alpha X \in \mathcal{K}(X), F_{\alpha}=F(X) \cap C_{\alpha}(X)$ is a subalgebra of $C^{*}(X)$ containing the ideal $C_{\mathrm{K}}(X)$ of all continuous functions with compact support.

Recall that $C_{\infty}(X)$ is the ideal of $C^{*}(X)$ consisting of all functions in $C(X)$ that vanish at infinity.

For $\alpha X \in \mathcal{K}(X)$, the map

$$
\psi: C_{\alpha}(X) \rightarrow C\left(K_{\alpha}(X)\right),\left.\quad f \mapsto f^{\alpha}\right|_{K_{\alpha}(X)},
$$


is a surjective morphism whose kernel is $C_{\infty}(X)$ (see $[5,2.4 .6]$ or $[17,5]$ ). Observe that, for $f \in F_{\alpha}(X), f^{\alpha}\left(K_{\alpha}(X)\right)$ is a finite set, and so $\left.f^{\alpha}\right|_{K_{\alpha}(X)} \in$ $\mathrm{LC}\left(K_{\alpha}(X)\right)$.

3.2. Theorem. For $\alpha X \in \mathcal{K}(X)$, the map

$$
F_{\alpha}(X) \rightarrow \mathrm{LC}\left(K_{\alpha}(X)\right),\left.\quad f \mapsto f^{\alpha}\right|_{K_{\alpha}(X)},
$$

is a surjective morphism whose kernel is $C_{\mathrm{K}}(X)$.

Proof. It is certainly an $\mathbb{R}$-algebra morphism whose kernel is $C_{\infty}(X) \cap$ $F_{\alpha}(X)=C_{\mathrm{K}}(X)$. In order to prove that it is surjective, it is enough to see that every idempotent of $\operatorname{LC}\left(K_{\alpha}(X)\right)$ has a predecessor in $F_{\alpha}(X)$. Given an idempotent $e \in \mathrm{LC}\left(K_{\alpha}(X)\right)$, let $g \in C(\alpha X)$ be an extension of the function $e$ to the whole $\alpha X$, and set

$$
h=3\left[\left(g \vee \frac{1}{3}\right) \wedge \frac{2}{3}\right]-1 .
$$

Finally, set $f=\left.h\right|_{X}$ and

$$
K=\left\{p \in \alpha X: \frac{1}{3} \leq g(p) \leq \frac{2}{3}\right\}
$$

$K$ is a compact subset of $X$, and $f(X-K) \subseteq\{0,1\}$. Therefore, $f \in F_{\alpha}(X)$. Moreover, $\left.f^{\alpha}\right|_{K_{\alpha}(X)}=e$.

3.3. Theorem. (a) If $\alpha X$ is a compactification of $X$ with $K_{\alpha}(X)$ zerodimensional, then $F_{\alpha}(X)$ is a subalgebra of $F(X)$ which contains $C_{\mathrm{K}}(X)$ and generates $\alpha X$.

(b) If $A$ is a subalgebra of $F(X)$ that contains $C_{\mathrm{K}}(X)$ and generates $\alpha X$, then $K_{\alpha}(X)$ is zero-dimensional and $A=F_{\alpha}(X)$.

Proof. (a) Since $K_{\alpha}(X)$ is zero-dimensional, $F_{\alpha}(X)$ generates a compactification of $X$ (see 2.2). Now it is enough to show that $F_{\alpha}(X)$ determines $\alpha X$. We show that $\left\{f^{\alpha}: f \in F_{\alpha}(X)\right\}$ separates points in $\alpha X-X$. Let $p_{1}$ and $p_{2}$ be two different points in $\alpha X-X$. Since $K_{\alpha}(X)$ is zero-dimensional, there is an idempotent $e \in C\left(K_{\alpha}(X)\right)$ such that $e\left(p_{1}\right) \neq e\left(p_{2}\right)$. According to $3.2, e=\left.f^{\alpha}\right|_{K_{\alpha}(X)}$ for some $f \in F_{\alpha}(X)$.

(b) It follows from Lemma 2.1 that $K_{\alpha}(X)$ is a zero-dimensional space, and certainly $A \subseteq F_{\alpha}(X)$. To prove the reverse inclusion it is enough to show that $A / C_{\mathrm{K}}(X)=F_{\alpha}(X) / C_{\mathrm{K}}(X)$. But this equality follows from 1.10 if we take into account the isomorphism $F_{\alpha}(X) / C_{\mathrm{K}}(X) \cong \mathrm{LC}\left(K_{\alpha}(X)\right)$ established in 3.2 .

For $X$ a Hausdorff locally compact space, $C_{\mathrm{K}}(X)$ separates points from closed sets in $X$, so that any subalgebra of $F(X)$ containing $C_{\mathrm{K}}(X)$ generates a compactification of $X$. 
3.4. Corollary. If $X$ a Hausdorff locally compact space, then the lattice $\mathcal{K}_{0}(X)$ of all compactifications of $X$ with zero-dimensional remainder is a complete lattice, which is isomorphic to the lattice $\mathcal{L}(\mathrm{LC}(\phi X-X))$ of all subalgebras of $\operatorname{LC}(\phi X-X)$.

$$
\text { Proof. } \mathcal{K}_{0}(X) \cong \mathcal{L}\left(F(X) / C_{\mathrm{K}}(X)\right) \cong \mathcal{L}(\mathrm{LC}(\phi X-X)) \text {. }
$$

Let $S_{\mathrm{e}}$ be the algebra of all eventually periodic real sequences, and let $S$ be the subalgebra of periodic sequences. Observe that

$$
\operatorname{Max}\left(S_{\mathrm{e}} / C_{\mathrm{K}}(\mathbb{N})\right) \cong \operatorname{Max} S,
$$

since $S_{\mathrm{e}}=C_{\mathrm{K}}(\mathbb{N}) \oplus S$. Hence, $S_{\text {e }}$ generates a compactification of $\mathbb{N}$ whose remainder is homeomorphic to the Cantor set (see 1.9). We get the same remainder (although not the same compactification) if we replace $S_{\mathrm{e}}$ by $C_{\mathrm{K}}(\mathbb{N})+S_{2}$.

3.5. TheOREM (compare with $[11]$ and $[9,2.1]$ ). Let $X$ be a Hausdorff locally compact space. Every compact metric space is a remainder of $X$ if and only if $F(X) / C_{\mathrm{K}}(X)$ contains a subalgebra isomorphic to $S_{2}$.

Proof. Assume that every compact metric space is a remainder of $X$. Then there is $\alpha X \in \mathcal{K}(X)$ such that $\alpha X-X \cong \operatorname{Max} S_{2}$. Since

$$
\operatorname{Max}\left(F_{\alpha}(X) / C_{\mathrm{K}}(X)\right) \cong \alpha X-X \cong \operatorname{Max} S_{2},
$$

it follows from 1.13 that $F_{\alpha}(X) / C_{\mathrm{K}}(X)$ is isomorphic to $S_{2}$. Let us prove the converse. On the one hand, it is well known that a compact Hausdorff space is a remainder of $X$ if and only if it is a continuous image of $\beta X-X$. On the other hand, every compact metric space is a continuous image of the Cantor set. Hence, it is enough to show that $X$ has a remainder homeomorphic to the Cantor set. By the hypothesis, there exists $A \in \mathcal{L}\left(F(X) / C_{\mathrm{K}}(X)\right)$ such that $A \cong S_{2}$. If $\psi: F(X) \rightarrow F(X) / C_{\mathrm{K}}(X)$ is the canonical morphism, then $\operatorname{Max} \psi^{-1}(A)$ is a compactification of $X$ whose remainder is Max $S_{2}$.

3.6. TheOrem. Let $X$ be a Hausdorff locally compact space, and let $\alpha X \in \mathcal{K}_{0}(X)$. The following conditions are equivalent:

(a) $\alpha X-X$ is a finite set.

(b) $F_{\alpha}(X) / C_{\mathrm{K}}(X)$ is a finite-dimensional algebra.

(c) $F_{\alpha}(X)=C_{\mathrm{K}}(X)+\mathbb{R}[f]$ for some $f \in F(X)$.

(d) $\alpha X$ is the compactification determined by a single $f \in F(X)$.

Proof. For the equivalence of conditions (a), (b) and (c), apply 1.11 to $F_{\alpha}(X) / C_{\mathrm{K}}(X)$. To see that (c) implies (d), observe that if $f \in F(X)$ and $F_{\alpha}(X)=C_{\mathrm{K}}(X)+\mathbb{R}[f]$, then $\alpha X$ is the compactification determined by $f$. Finally, let $\alpha X$ be the compactification determined by a single function $f \in$ $F(X)$. The function $\left.f^{\alpha}\right|_{\alpha X-X}$ is injective and has finite range. Therefore, $\alpha X-X$ is finite. This proves that (d) implies (a). 
3.7. Corollary. Let $X$ be a Hausdorff locally compact space. The lattice of all compactifications of $X$ with finite remainder is a lower complete lattice, which is isomorphic to the lattice of all finite-dimensional subalgebras of $\mathrm{LC}(\phi X-X)$.

3.8. Corollary (Woods [23]). For $X$ and $Y$ Hausdorff locally compact spaces, the following conditions are equivalent:

(a) The remainders $\phi X-X$ and $\phi Y-Y$ are homeomorphic.

(b) The lattices $\mathcal{K}_{\mathrm{F}}(X)$ and $\mathcal{K}_{\mathrm{F}}(Y)$ are isomorphic.

(c) The lattices $\mathcal{K}_{0}(X)$ and $\mathcal{K}_{0}(Y)$ are isomorphic.

Proof. This follows from 1.13, 3.4 and 3.7 .

3.9. Corollary (Nowiński [18]). If $X$ is a Hausdorff locally compact space, then $\phi X$ is the least upper bound of all compactifications of $X$ with finite remainder.

Proof. We denote by $\alpha_{f} X$ the compactification determined by a single function $f \in C^{*}(X)$. If $\alpha X$ is a compactification with finite remainder, then $\alpha X \leq \phi X$, since $\phi X$ is the maximum compactification of $X$ with zerodimensional remainder. Let $\gamma X$ be a compactification such that $\gamma X \geq \alpha X$ for every compactification $\alpha X$ with finite remainder. In particular, $\gamma X \geq$ $\alpha_{f} X$ for every $f \in F(X)$. Therefore, $C_{\gamma}(X) \supseteq F(X)$, and so $\gamma X \geq \phi X$.

\section{References}

[1] M. F. Atiyah and I. G. Macdonald, Introduction to Commutative Algebra, AddisonWesley, London, 1969.

[2] B. J. Ball and S. Yokura, Compactifications determined by subsets of $C^{*}(X)$, Topology Appl. 13 (1982), 1-13.

[3] —, Compactifications determined by subsets of $C^{*}(X)$, II, ibid. 15 (1983), 1-6.

[4] R. Dickman and R. McCoy, The Freudenthal compactification, Dissertationes Math. 262 (1988).

[5] J. M. Domínguez, Álgebras de funciones continuas y sus espacios de ideales maximales, Ph.D. thesis, Univ. of Valladolid, 1983; MR 85g:46089.

[6] - Compactificaciones con resto 0-dimensional, Actas X Jornadas Hispano-Lusas de Matemáticas, Sección VII, Univ. of Murcia, 1985, 101-105; MR 87k:54037.

[7] N. D. Filippov, Projectivity of lattices, Amer. Math. Soc. Transl. 96 (1970), 37-58.

[8] L. Gillman and M. Jerison, Rings of Continuous Functions, Springer, New York, 1978.

[9] J. Hatzenbuhler and D. A. Mattson, Spaces for which all compact metric spaces are remainders, Proc. Amer. Math. Soc. 82 (1981), 347-350.

[10] - - - Spaces between $X$ and its Freudenthal compactification, Period. Math. Hungar. 31 (1995), 27-31.

[11] J. Hatzenbuhler, D. A. Mattson and W. S. Sizer, An algebraic characterization of remainders of compactifications, Canad. Math. Bull. 26 (1983), 347-350. 
[12] M. Henriksen, An algebraic characterization of the Freudenthal compactification for a class of rimcompact spaces, Topology Proc. 2 (1977), 169-178.

[13] - Rings of continuous functions from an algebraic point of view, in: Ordered Algebraic Structures, J. Martinez (ed.), Kluwer, Dordrecht, 1989, 143-174.

[14] J. R. Isbell, Uniform Spaces, Amer. Math. Soc., Providence, 1964.

[15] K. Keimel, Algèbres commutatives engendrées par leurs éléments idempotents, Canad. J. Math. 22 (1970), 1071-1078.

[16] K. D. Magill, Jr., The lattice of compactifications of a locally compact space, Proc. London Math. Soc. 18 (1968), 231-244.

[17] F. Mendivil, Function algebras and the lattice of compactifications, Proc. Amer. Math. Soc. 127 (1999), 1863-1871.

[18] K. Nowiński, Closed mappings and the Freudenthal compactification, Fund. Math. 76 (1972), 71-83.

[19] D. Sachs, The lattice of subalgebras of a Boolean algebra, Canad. J. Math. 14 (1962), 451-460.

[20] O. Stefani and A. Zanardo, Alcune caratterizzazioni di una sottoalgebra di $C^{*}(X)$ e compattificazioni ad essa associate, Rend. Sem. Mat. Univ. Padova 53 (1975), 363-367.

[21] Y. Ünlü, Spaces for which the generalized Cantor space $2^{J}$ is a remainder, Proc. Amer. Math. Soc. 86 (1982), 673-678.

[22] E. Wajch, Subsets of $C^{*}(X)$ generating compactifications, Topology Appl. 29 (1988), $29-39$.

[23] R. G. Woods, Zero-dimensional compactifications of locally compact spaces, Canad. J. Math. 26 (1974), 920-930.

Departamento de Álgebra, Geometría y Topología

Facultad de Ciencias

Universidad de Valladolid

47005 Valladolid, Spain

E-mail: jmd@agt.uva.es

Received 26 August 2002;

in revised form 10 July 2003 